\title{
ALGUNAS CONSIDERACIONES EN TORNO AL VOCABULARIO Y AL DICCIONARIO DE ELIO ANTONIO DE NEBRIJA
}

\section{INTRODUCCIÓN.}

Existe consenso entre los lingüistas y filólogos acerca de la extraordinaria importancia que la obra de Elio Antonio de Nebrija ha tenido para la filología hispánica (cfr. BAHNer, 1966: 41) y, sin embargo, los problemas que hay pendientes sobre su persona y obra son todavía numerosos, partiendo del hecho mismo de su nacimiento (cfr. Quilis en Nebrija, 1980: 9-10). Porque un detalle en apariencia tan poco importante como que naciera en 1444, como suele admitirse corrientemente (cfr. BAHNER, 1966: 48; BeLlido, 1945 : 117-118, y Olmedo, 1942: 9-10), o que viniera al mundo en 1441, sirviéndome de los datos biográficos que el propio Nebrija resume en su Vocabulario ${ }^{1}$, no queda sin consecuencias para algo tan importante como lo es la fecha de publicación de esta obra. Si se acepta la de 1444, el Vocabulario se publicaría en 1495, año que suele admitirse por los investigadores; si hubiera nacido en 1441, este repertorio se habría publicado al mismo tiempo que la Gramática de la lengua castellana y el Diccionario, es decir, en 1492.

Pero estos pequeños problemas pendientes no deben ocultarnos el puesto de honor que el profesor salmantino tiene en la ciencia del lenguaje: fue un adelantado de las descripciones fonéticas (Alonso, 1949: 3) en sus Reglas

1 Llamaremos Vocabulario:EL al Dictionarium ex hispaniensi in latinum semonem, es decir, al Vocabulario español-latino; y Diccionario:LE al Lexicon ex sermone latino in hispaniensem, es decir, al Diccionario latino-español. 
de orthografía en la lengua castellana, en 1517 ; publicó la primera Gramática de la lengua castellana en 1492, con anterioridad a la primera italiana y francesa (Quilis en Nebrija, 1980: 80) e hizo realidad el primer Vocabulario español-latino, pues si bien en 1490 había aparecido el Universal vocabulario de Alfonso de Palencia -latino-español solamente-, éste se quedó anticuado en relación con la modernidad que presenta el de Nebrija, como trataré de mostrar más adelante (cfr. Colón-Soberanas en Nebrija, 1979: 24).

No voy a ocuparme aqui de aspectos tan importantes de la obra de $\mathrm{Ne}$ brija como son los anteriores. Mi fin es mucho más modesto: pretendo analizar algunos aspectos, como el intento de Nebrija de regularizar la grafía del castellano, para pasar después a examinar la idea generalizada sobre la falta de arabismos en el Vocabulario y finalmente a pronunciarme sobre la ausencia de marca dialectal en sus obras lexicográficas, que suele aceptarse unánimemente.

Para ello empezaré comparando el Diccionario (edición de Germán Colón y Amadeu-J. Soberanas, Barcelona, Puvill, 1979) y el Vocabulario (edición facsímil de la Real Academia Española, Madrid, 1951) ${ }^{2}$. Me referiré brevemente a sus rasgos lingüísticos, a la forma como Nebrija realizó estos léxicos y a la diferente perspectiva en que se sitúa cada uno de ellos. En segundo lugar estudiaré la falta de nivelación gráfica en el Diccionario y en el Vocabulario. En el último apartado mostraré la modernidad de la obra lexicográfica de Nebrija respecto a otros diccionarios de su época y de los datos léxicos proporcionados por el profesor salmantino, que no habiendo sido todavía utilizados, abren nuevas perspectivas a nuestros conocimientos de la historia de algunas palabras españolas, muy especialmente en lo referente a la presencia del arabismo en estas dos obras.

\section{Estudio comparativo entre el Vocabulario y el Diccionario.}

Nebrija fue también deudor de su siglo y por ello refleja en sus obras lexicográficas las vacilaciones de la lengua de su época (cfr. LAPESA, 1980: 279-280, y QUiLIs, 1977 : 84-85). Esto es algo que no debemos olvidar cuan-

3 G. Colón y A. J. Soberanas reprodujeron en facsimil el ejemplar de la Biblioteca Universitaria de Barcelona para el Diccionario y la Real Academia Española reprodujo la edición príncipe del Diccionario hispano-latino de Salamanca para el Vocabulario. Acerca de éstas y otras ediciones, véase: ANTonio OdriozolA, "La caracola del bibliófilo nebrisense", Revista de Bibliografia Nacional, VII, 1946, págs. 24-33; el Prólogo citado de G. Colón y A. J. Soberanas, págs. 34-36, y el Prólogo que la Real Academia hizo al Vocabulario español-latino. 
do solemos hablar del gramático de Lebrixa acudiendo a lo que dice de su intento de uniformar gráficamente el español, olvidándonos de lo que hace (Rosenblat, 1951 : XVII-XVIII).

No faltan en sus repertorios vacilaciones fonéticas corrientes a finales de la Edad Media, como $^{\mathbf{3}}$ :

$e \sim i:$ adevinar:LE / adivinar:LE, EL; adevino:LE / adivino:LE, EL. arrexaque:LE, EL / arrixaque:LE.

decender:LE / decendir:EL.

emperial:EL / imperial:EL.

entredezir:LE / entredizir:EL.

espremir:LE, EL / esprimir:LE.

gengibre:LE, EL / gingibre:LE.

hediondo:LE, EL / hidiondo:LE, EL.

imagen:LE, EL / imágines:L.E, EL.

redemir:EL / redimir:LE.

rencón:LE / rincón:LE, EL.

requerir: $\mathrm{EL} /$ requirir:LE.

restreñir:LE / restriñir:EL.

reteñir:LE / retiñir:LE, EL.

texedor:LE / texidor:EL.

vanedad:LE, EL / vanidad:LE.

$o \sim u$; abondar:LE, EL / abundar:LE.

acochillar:LE / acuchillar:EL.

cobrir:LE, EL / cubrir:LE, EL.

escopir:LE, EL / escupir:LE.

instromento:LE / instrumento:LE, EL.

mormullo:LE / murmullo:EL.

onción:LE / unción:LE, EL.

resolución:LE, EL / resulución:EL.

$a \sim e:$ ascaravajo:LE / escaravajo:LE, EL.

luziérnaga:LE, EL / luziérnega:LE.

maxilla:LE, EL. / mexilla:LE, EL.

Nagroponte:LE / Negroponte:LE.

parlasia:LE / perlesia:LE, EL.

Tampoco faltan las vacilaciones fonéticas en el consonantismo, como en el caso de la f-inicial (feno:L.E, EL / heno:LE; finiestra:LE / hiniestra:LE, EL; fossario:EL / hossario:LE, EL); en los grupos $b+l \sim b+r$ (roble:LE, $\mathrm{EL} /$ robre:LE), $l+$ cons. $\sim r+$ cons. (alçapiélago:LE / arçapiélago:LE,

- LE = Diccionario; $\mathrm{EL}=$ Vocabulario. 
EL) o en la utilización de $b$ y $v$ (turbar:EL / turvar:LE, EL; abispa:LE, EL; taverna:LE, EL; bever:LE, EL; cavallo:LE, EL; carvón:LE, EL; carvonero:LE, EL; valadi:EL), y en algunos casos más de las sibilantes $(c \sim z:$ cenceño:EL / cenzeña:LE; ss $\sim s:$ apressurarse:LE / apresurarse: LE; $s \sim x$ : casquete:EL / caxquete:LE; $j \sim x$ : cañaheja:LE / cañahexa: LE, EL).

Partamos de este hecho, para no convertir desde el principio lo que se predica en lo que se hace y para poder, por tanto, entender la modernidad en los plantamientos de nuestro gramático, aunque esa modernidad no la cumpla sistemáticamente en sus escritos.

La vacilación que se percibe en la obra de Nebrija debe ser precisada en cuanto a las diferencias que muestran sus dos grandes creaciones lexicográficas: el Vocabulario y el Diccionario.

No me voy a parar en los criterios que utiliza Nebrija en la elaboración de estas obras, pues algunos de éstos ya han sido estudiados por A. Quilis (1980: 59-61). Ahora bien, antes del sucinto estudio contrastivo quiero mostrar las diferentes concepciones que presiden la construcción de estos dos léxicos. G. Colón y A. J. Soberanas (1979: 10) han indicado que la ordenación del Vocabulario está pensada a partir del castellano, y la del Diccionario lo está desde el latín, a lo que voy a añadir otros aspectos que confirman esta teoria, aparte de plantear algunas cuestiones que chocan con las fáciles soluciones dadas tradicionalmente por otros estudiosos.

Creo que Nebrija al llevar a cabo su tarea lexicográfica tuvo un planteamiento diferente para cada obra, a pesar de que en algunos aspectos aprovechase las mismas técnicas para las dos; los motivos que me animan a defender esta teoría son los siguientes:

El Diccionario se sitúa en un plano latino y helenista, y si queremos extranjerizante, por lo que se refiere a los nombres propios. En él figuran gran cantidad de nombres que no están en el Vocabulario, pero además se trata de nombres de emperadores, reyes, guerreros, héroes, dioses, filósofos, pintores, médicos latinos y griegos, nombres de la mitología de estos pueblos y otros nombres de pueblos extranjeros; por el contrario, son muy escasos los nombres de reyes, héroes, emperadores españoles. La grafía de esos nombres, en muchos casos, es la misma que la latina o cercana a ella, por el mantenimiento de grupos cultos latinos. Algunos de ellos son los siguientes: Abdera, Achaia, Achilles, Alcmeón, Althea, Amphión, Amithaón, Antiochía, Apelles, Archiloco, Loth, Ptolemeo, Polus, Rachel, Rebecca. Ninguno de los relacionados figura en el Vocabulario.

Los nombres propios que presenta el Vocabulario se refieren a "astrólogos moros" (como los cita Nebrija), ciudades y nombres españoles; y na- 
turalmente la grafía es la española, así Agustín, Albumasar, Alcabicio, Alcántara, Almeria, Almuñécar, Alonso, Alvarez, Ana, Antolín, Lázaro, Leonor, López, Llerena, Rodrigo, Úbeda, Uesca. Algunos pocos se refieren a Francia, Italia y Portugal como Arle, Assis, Aviñón, Rávena. Los nombres latinos y griegos prácticamente están ausentes. Todos los que he citado y muchos más no se hallan en el Diccionario.

Además, los nombres propios en el Vocabulario no suelen romper el orden alfabético: así, Andrés está entre andén y anegar; Rodrigo entre rodilla y Rodriguez; Lucas entre Lucano y lucha; Roán entre risco y roanesa; no obstante, en algún caso si existe esta ruptura como en Leonardo entre leonado y León. En el Diccionario se rompe mucho más frecuentemente el orden alfabético: Acrisius está detrás de acriter; Caligula antes de caligo; Electra detrás de electrinus; Hamilcar detrás de Hamulus; Tyresias detrás de Tyridates. Lo que parece indicar que en el Vocabulario estos nombres fueron introducidos desde el principio, mientras que en el Diccionario no había tenido la intención de incluirlos junto al léxico común, motivo por el que falla el orden alfabético.

En segundo lugar, el campo semántico religioso es diferente en estas dos obras. El Vocabulario ofrece un mayor número de nombres religiosos, de la cristiandad: abadia, angelical, arçobispal, demonio, letania, reliquiario, resurreción, etc., voces que no figuran en el Diccionario. La terminología de éste es, por el contrario, más pagana, filosófica, científica: afeitadura de muger, albriciar, alcautería, arismética, arteria, deletación, legitimación, libertar, lira, racional, léxico que no se encuentra en el Vocabulario.

En tercer lugar, la estructura es diferente en los dos: así por ejemplo, en el Diccionario constantemente hace referencia a la entrada o entradas anteriores, con lo cual se echan en falta adjetivos, formas adverbiales y derivados de la entrada a la que hace referencia, o sinónimos. Tal es el caso de infandus, a, um 'por cosa no de hablar', infandum 'por aquello mesmo'; lamentor, aris 'por llorar con bozes', lamento, as 'por aquello mesmo', lamentatio, onis 'por aquel lloro', lamentum, $i$ 'por aquello mesmo'; lingulaca, ae 'por parlero', linguax, acis 'por aquello mesmo'; suspiratio, onis 'por sospiro', suspiratus, a, um 'por aquello mesmo'.

En el Vocabulario, al partir de los términos españoles, busca la forma correspondiente que quiere incluir, ya sea un adjetivo, adverbio o sustantivo: esquina, esquinada cosa, esquinancia; estable, establemente, establecer, establecimiento; quebrar, quebradura, quebrantable, quebrantador.

Finalmente, el Vocabulario contiene muchos más arabismos que el Diccionario; hecho normal si se piensa en la perspectiva española con que está planteado. Ofrece el Diccionario también muchas más variantes y vacilacio-

LXVII, $1 .^{\circ}-2 .^{\circ}-7$ 
nes que el Vocabulario ", lo cual no es de extrañar, dado que en éste el léxico español sigue un orden alfabético, a diferencia de lo que en lo referente a los vocablos de nuestra lengua ocurre en el Diccionario, por aparecer estas voces sólo como traducciones de otras latinas. Ello explica que en este último caso utilice unas veces una variante y otras veces otra : administrar / aministrar, Apollo / Apolo, etc. Pero sobre estos temas volveré más adelante.

No me resisto a dejar planteadas algunas cuestiones importantes que merecen el que algún día se les encuentre una solución. Éstas son:

¿Cómo si el Diccionario es anterior al Vocabulario - según dice Isabel Acero- ${ }^{5}$ no figuran en el Vocabulario muchos vocablos que se encuentran en el Diccionario, como acabador, acicalador, acompañable, acostumbradamente, adoptación, afeamiento, ahorrar, alboroto, almizquera, alquilar, amansable, animación, aparcero, apostar, arroz, asperidad, avezilla, dador, deleznablemente, dentadura, derivación, desacostumbrar, desagradamiento, latón, lexura, libertar, lícito, locamente, rebelión, reboltosa, rendición, renovero, resonar, rostro, ulceración, untaza, etc. ?

Por otra parte, habría que matizar la afirmación de G. Colón y A. J. Soberanas (1979: 11) acerca de que las adiciones en la edición hispano-latina, es decir, en el Vocabulario, son relativamente escasas. Es esta una impresión que no se ve confirmada cuando se acude a recuentos llevados a cabo con ordenador. De los recuentos llevados a cabo en las letras $a, d, l, l l, r$ y $u$ los resultados son los siguientes: en el Diccionario se encuentran 689 términos que no se hallan en el Vocabulario, de los cuales 509 son nombres comunes y 180 son nombres propios, repartidos de esta manera: voces correspondientes a la $a: 222$. a la $d: 152$, a la $l: 41$, a la $l l$ : 5 , a la $r: 76$ y a la $u: 13$; nombres propios concernientes a la $a: 103$, a la $d: 27$, a la $l: 35$, a la $r: 11$ y a la $u: 4$. En el Vocabulario figuran 1.439 vocablos que no aparecen en el Diccionario, nombres comunes 1.389 y nombres propios 50, distribuidos de esta forma: dicciones correspondientes a la $a$ : 605 , a la $d: 424$, a la $l: 107$, a la $l l: 6$, a la $r: 230$ y a la $u: 17$; nombres propios pertenecientes a la $a: 23$, a la $d: 3$, a la $l: 12$, a la $r: 8$ y a la $u: 2$. Como se habrá comprobado, las entradas en el Vocabulario son mucho más numerosas, con la excepción de los nombres propios. Pero en el cómputo

4 Véase cómo el número de variantes es superior en el Diccionario en los ejemplos citados anteriormente al hablar de las vacilaciones fonéticas de la Edad Media.

5 "Según las fechas que acabamos de mencionar, sabemos que Nebrija redactó primero la parte latina y que posteriormente con la trasposición de ese material redactó la parte castellano-latina", en "El Diccionario latino-español y el Vocabulario españollatino de Elio Antonio de Nebrija: análisis comparativo", Anuario de Lingüistica Hispánica, I, 1985, pág. 12. 
total el número de términos agregados es un poco más del doble en el $V o$ cabulario frente al Diccionario.

Ahora bien, a pesar de que en estas cinco letras se encuentran muchos más vocablos españoles en el Vocabulario, esto no quiere decir que Nebrija no los conociera a la hora de redactar el Diccionario, y que, por lo tanto, pudiera ser un indicio para señalar que el Vocabulario fue redactado con posterioridad; sino que como la perspectiva del Diccionario es latina, su autor no tiene ningún empacho en hacer referencia a las entradas anteriores ". Lo que a Nebrija le interesa en este Diccionario es el léxico latino, y no tratar de ser exhaustivo con el vocabulario español. No sería creíble que resquebrajarse no lo conociera cuando si introduce resquebrajadura; apareciendo reliquias, retorcer, ablandar y desobediencia, es extraño que no conociera reliquiario, retorcedura, ablandadura, desobedecer y despedaçadura, que sí figuran en el Vocabulario; y, contrariamente, retorcido, despedaçable y despedaçamiento sólo están en el Diccionario.

Finalmente, si la composición del Diccionario fue anterior, ¿ cómo el $V o$ cabulario presenta errores que no se hallan en aquél ? Es raro que la segunda obra lexicográfica ofrezca más errores en un léxico que ya figura en la primera como: solemnemente 'solemnitas, celebritas', cuando lo correcto y lo que se encuentra en el Diccionario es 'solemniter, celebriter'; lo mismo sucede con orinal 'matela', siendo lo correcto 'matella'; limpieza 'mondicia' en lugar de 'mundicia'; engañar apartando 'seductio, onis' en vez de 'seduco, is'; deslizadero 'vide deleznadoro' cuando debería decir 'deleznadero'; aguatadora cosa en lugar de aguaitadora; enveramiento de nervios en vez de envaramiento. Contra esto se me podrá replicar que tales errores son debidos a los impresores; pero ¿no hemos de suponer que Nebrija al escribir el Diccionario tuviera las fuentes de este léxico delante?, motivo por el que no comete errores, mientras que, por el contrario, el Vocabulario pudo ser redactado de memoria, lo cual explicaría bien los fallos latinos. No es esta una posibilidad que debamos descartar sin más.

Por lo que se refiere al estudio contrastivo de estas dos obras, hay coincidencias y diferencias que no carecen de interés ${ }^{7}$ :

- En el Diccionario la referencia a la entrada anterior es en muchos casos completa, como en forsan, forsitan 'por aquello mesmo', en los dos; mientras que en el Vocabulario, a pesar de que a veces también hay referencias a las entradas, éstas, sin embargo, son siempre parciales, así oficial desta manera, pensamiento assi, etc.

7 Soy consciente de que para hablar de posibles características catalanas, gallegoportuguesas, etc., habría que hacer un estudio exhaustivo de todo el léxico del Diccionario y del Vocabulario; no obstante, voy a adelantar los datos que he encontrado en un primer rastreo, a pesar de que éstos puedan resultar arriesgados. 
1. El Diccionario presenta mayor número de grafías cultas y de cultismos que el Vocabulario. Debido probablemente al hecho de que en aquella obra está traduciendo del latín. Ello explica que se le escapen grafías latinas, como he señalado ya en los nombres propios, pero también en muchos otros casos, como legislator, pinctor, postponer, solemnidad, auctor...

2. El Diccionario posee algunos rasgos fonéticos en los que quizá pudiera verse un influjo del catalán. Se trata de vacilaciones entre la $a$ y $e$ átonas: ascaravajo, aspumosa, astar 'estar', confacionada, da 'de', despadaçar, generalmante, paqueña, no, partas y entre la $e$ y $a$ átonas: cose 'cosa', die, cavelleros, equél, equello, merchitable. Claro está que estos hechos - si es que no tienen otra explicación - se debería a la procedencia del impresor.

3. El léxico andaluz y los arabismos están mucho mejor representados en el Vocabulario que en el Diccionario - ya he señalado que debido a la perspectiva española en la que se sitúa el Vocabulario-. Encuentro en aquél términos como almirón, chicarro 'cigarra' y alarifadgo, alaxur, etc.

4. Igualmente se encuentran algunos rasgos gráficos gallego-portugueses -si es que no son meras erratas- : cono 'coño', vina, que faltan, en cambio, en el Diccionario. En cuanto a los occidentalismos y leonesismos se reparten en las dos obras lexicográficas, pero no siempre coinciden en las voces empleadas: así, lamber figura solamente en el Diccionario (lamer en EL / LE) y lóbado en los dos. Todo ello nos lleva -aunque con tantas precauciones como en el caso de los catalanismos- a suponer que el impresor tuviera un origen gallegoportugués.

5. Curiosamente, el Vocabulario contiene vocablos de carácter arcaico que no aparecen en el Diccionario y cuya última documentación en algunos casos es Nebrija; particularidad que contrasta con la datación que se ha dado del Vocabulario. Algunas de estas voces son afruenta, aprés, atijara, llantear, rogarias.

Finalmente, el número de variantes dobles es más elevado en el Diccionario que en el Vocabulario $^{8}$. Ya he explicado que se debe a que en el Diccionario no existe el control del orden alfabético, que hubiera permitido con más comodidad una normalización gráfica.

8 Véase la nota 4, así como las vacilaciones gráficas y fonéticas estudiadas en este trabajo. 
2. Fallido intento de Nebrija en la regularización de la grafía CASTEllana.

Es visible la falta de nivelación gráfica en el Diccionario y en el Vocabulario. Valdés en el Diálogo de la lengua negó a Nebrija la capacidad de regularizar el castellano, por su condición de andaluz (BAHNER, 1966: 69), a pesar de que el Antonio en el Prólogo a su Gramática de la lengua castellana había expresado su deseo de estabilizar la lengua vulgar de España y de fijar el uso del castellano: "Hasta nuestra edad anduvo suelta e fuera de regla, e a esta causa a recebido en pocos siglos muchas mudanças" (QuiLIS en NebriJa, 1980: 81).

Los críticos nebrisenses actuales como W. Bahner, A. Quilis, E. de Bustos, al referirse a la Gramática de la lengua castellana y a las Reglas de orthografía en la lengua castellana, concluyen en que el intento de Nebrija en estas obras fue el de fijar el uso del español y estabilizar la lengua vulgar de España, regularizando el uso gráfico del castellano ${ }^{9}$; aunque W. Bahner se da cuenta, sin embargo, de que "lo estéril de tal intento en aquella época lo demuestra el hecho de que los rasgos fundamentales del castellano no se impusieron hasta el siglo xvi" (1966: 44). Menéndez Pidal se había dado cuenta también de que "Nebrija no tuvo presente la distinción de una lengua viva y una muerta" (1950: 23).

Más arriba he mostrado algunos ejemplos de vacilación que demuestran que el propio Nebrija fue incapaz de llevar a la práctica su deseo normalizador. Veamos ahora algunas vacilaciones más, en lo gráfico, que no tienen nada que ver con las variantes de pronunciación del castellano cuatrocentista y que se relacionan exclusivamente con usos puramente de escritura.

La vacilación gráfica la tenemos en ${ }^{10}$ :

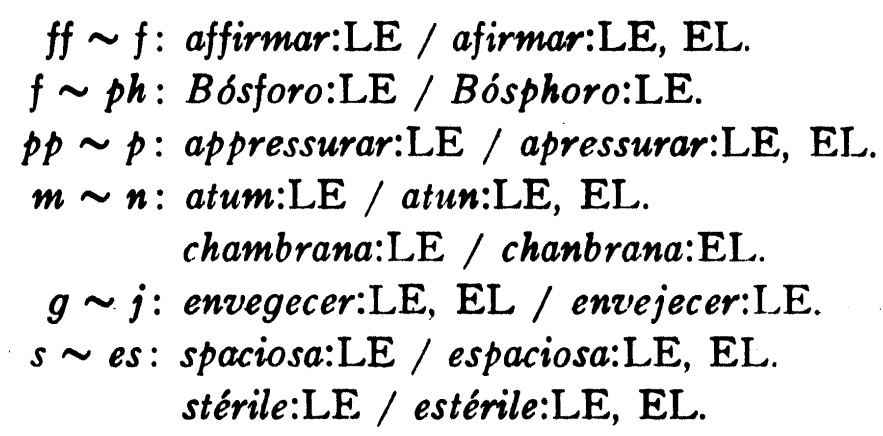

- La opinión de E. DE Bustos en "Nebrija, primer lingüista español", Academia Literaria Renacentista: Nebrija y la Introducción del Renacimiento en España, Salamanca, 1983, págs. 211 y 218, es que Nebrija intentó regularizar la grafía del castellano.

10 Excepto en algún caso, no cito más que uno o dos ejemplos de cada vacilación. 


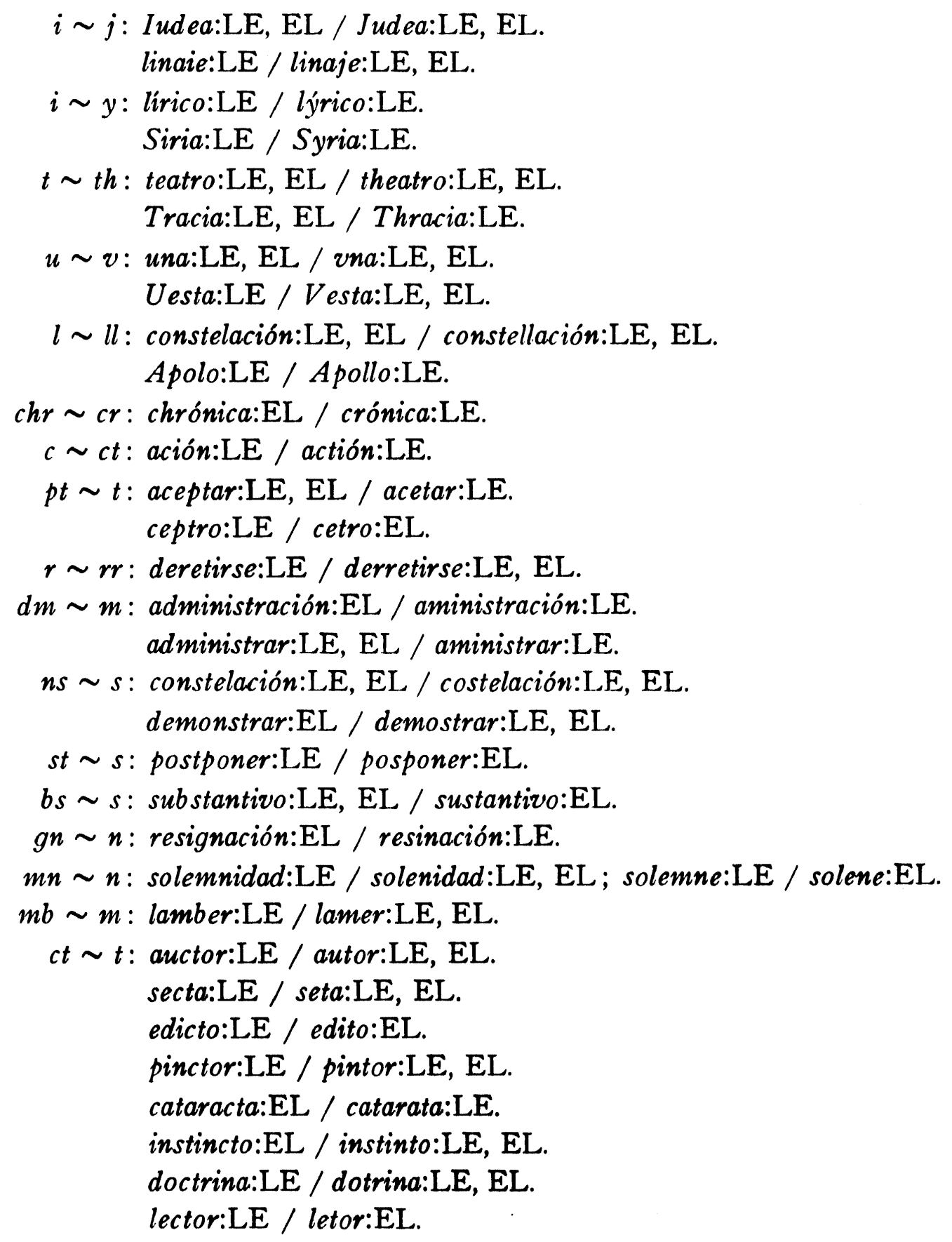

Señalemos de paso que en el plano morfológico aparecen también algunas vacilaciones: árbol conocida:LE, EL y árbol olorosa:LE, EL frente a árbol conocido:LE, EL y árbol oloroso:LE. Arbol con género femenino es característico del leonés.

Como se podrá comprobar, la vacilación abarca tanto a los nombres comunes como a los propios, siendo las variantes más cultas y latinizantes las que suelen hallarse en el Diccionario. $\mathrm{Y}$ estas vacilaciones, tanto las fonéticas 
(de la que hablé al principio) como las gráficas son las típicas de los escritores del xv. No voy a insistir aquí en el hecho ya señalado de la mayor inestabilidad, en cuanto a la norma, en el Diccionario que en el Vocabulario. La razón de esto ya la he indicado: la ordenación alfabética del léxico castellano en el Vocabulario frente a la libertad de uso que permite el Diccionario: esta libertad es la que origina que Antonio de Nebrija coloque espaciosa y spaciosa en distintos lugares (espaciosa figura en la entrada 28.219 y spaciosa en la 25.811).

Nebrija tiene un puesto de honor en la "normalización" gráfica del español; pero lo tiene fundamentalmente por el rigor de su doctrina, no por el cumplimiento de sus propios preceptos. Con todo, hay un punto más de coherencia gráfica en su obra que en la de otros escritores españoles del siglo xv; pero aun en eso no debemos olvidar el hecho que separa a Nebrija de un Santillana, un Mena o un Villena: la mediación de la imprenta y la necesidad de los impresores de ir más lejos en la normalización gráfica de lo que lo hacían quienes antes de la existencia de la imprenta podían permitirse todas las libertades en este terreno.

\section{Modernidad de la obra lexicográfica de Nebrija. La presencia DEL ARABISMO.}

La modernidad de los léxicos de Nebrija ya ha sido señalada por G. Colón y A. J. Soberanas (1979: 24). Su obra posee una extraordinaria importancia para la historia de la filología española. El Diccionario y el Vocabulario fueron una empresa monumental para su tiempo, como ha indicado Julio Casares (1947: 335); y cuyo mérito -como analizaré a continuación- no disminuye porque viera la luz dos años después del Universal vocabulario de Alfonso de Palencia, magnífico como documento lingüístico, pero que no contiene, sin embargo, más términos castellanos que los necesarios para traducir el texto latino de la columna que tiene al lado. Lo de menos en esta obra es el léxico: lo que importa en ella, más que otra cosa, son las explicaciones de carácter enciclopédico que pudieran interesar a una persona culta del siglo $\mathrm{xv}$.

Aunque la obra lexicográfica nebrisense tampoco está completamente libre de este defecto. Bastará con señalar la importancia de los nombres propios, sobre todo en el Diccionario, para que sobre cualquier otra argumentación ${ }^{11}$.

11 Hay otros muchos rasgos típicos de los diccionarios enciclopédicos, como es el caso de los sinónimos separados por $o$, ya por $e$, bien por $i$, o por por, en lo que $\mathrm{Ne}$ brija es deudor de una actitud tradicional. A. REY, en Encyclopédies et dictionnaires, 
Desde un punto de vista teórico, los dos léxicos de Nebrija no son auténticos diccionarios, sino una mezcla entre enciclopedia y diccionario. Lo cual es perfectamente explicable, situándonos en la época en que fueron redactados. En comparación con los trabajos de lexicografía de su tiempo, no puede existir duda en cuanto a su modernidad.

Si confrontamos el Universal vocabulario de Alfonso de Palencia con los del profesor salmantino, se observará que mientras en el de aquél cada término es explicado, en numerosas ocasiones, por medio de divagaciones, y a veces con excesivo lujo de detalles, en los de Nebrija, por el contrario, su actitud principal es la concisión, la brevedad y el intento de sintetizar. Aunque a veces se olvide de estos preceptos y acuda a explicaciones, siempre breves. Cotéjese en los términos que a continuación relaciono la diferencia entre los dos lexicógrafos:
Adonay: $\quad \mathrm{P}^{12}$ 'es nombre del señor en la lengua delos hebreos el qual nombre se interpreta señor general mente'.
$N^{18}$ 'uno delos diez nombres de dios'.
Anguilla: $\quad \mathrm{P}$ 'se dize de angue que es culuebra que es semeiante a ella o por que mientra con mayor fuerça se aprieta tanto más presto se escabulle dela mano'.
$\mathrm{N}$ 'por el anguilla'.
Animadversio: $\mathrm{P}$ 'es vengança e sentençia algund reo ab animadverto el qual verbo también significa pensar'.
$\mathrm{N}$ 'por aquel castigo'.
Arra: $\quad$ P 'es dicha dela cosa por que se da no por que ella se aya de retornar como prenda mas por que ella perma- neçiendo todas las otras cosas se cumplan'.

\footnotetext{
Que sais-je?, París, 1982, pág. 19, es explícito en cuanto a la explicación que se puede dar a estos rasgos: "Une différence fondamentale entre l'encyclopédie et le dictionnaire tient à la nature des entrées. L'article d'encyclopédie, qui a pour objet la description du monde, utilise l'entrée comme un accès au domaine à décrire. Cette entrée n'est pas imposée comme signe, mais seulement comme contenu. Au contraire le dictionnaire, qui se propose la description des mots, présente des entrées qui sont l'objet même dont on parle en tant que signe; la nomenclature d'ensemble est imposée et chaque entrée, qui est envisagée dans sa forme est déterminée : on ne peut lui substituer aucun synonyme ni aucune traduction. Quant aux noms propres, qui figurent à la nomenclature des encyclopédies, le dictionnaire de langue les exclut. Néanmoins, il existe des dictionnaires, qui les prennent comme objets d'étude (dictionnaires toponymiques, onomastiques, étymologiques des noms propres)."

$12 \mathrm{P}=$ Alfonso de Palencia, Universal vocabulario en latin y en romance, Reproducción facsimilar de la edición de Sevilla, 1490, 2 tomos, Gráficas Cóndor, Madrid, 1967.
}

18 N = Antonio de Nebrija, Diccionario. 
Arrae: $\quad \mathrm{N}$ 'por las arras e señal dela compra'.

Cetra : $\quad \mathrm{P}$ 'es escudo fecho de cuero e que se doblega siendo guarnido de tres doblezes de cuero de búfalo o de buey o de otros animales que tienen el lomo del cuero muy rezio e tal que de ligero nonle passan daraga'.

$\mathrm{N}$ 'por el adarga de cuero'.

Dolosus: $\quad \mathrm{P}$ 'assechador por que engaña e para engañar asconde su maliçia e compone las con blandas palabras esto mesmo significa dolus dicho de fallendo ca tiene en sy ascondido el cuchillo para ferir'.

$N$ 'por cosa engañosa'.

Genus: $\quad \mathrm{P}$ 'es nascimiento y ensanchamiento dela casa e comienço que se dize de engendrar e segund ...'.

$\mathrm{N}$ 'por el linaje de cada cosa'.

Mandragora: $\mathrm{P}$ 'es un linaie de yerva cuyo fructo es semeiante a popón o melón pequeñito e llaman lo los latinos mançana dela tierra tiene la rayz a forma de ombre e por esto se llama antropomofeos ...'.

$\mathrm{N}$ 'por la mandrágora ierva'.

Monile: $\quad \mathrm{P}$ 'es adornamiento de muger o de cavallos desdel cuello pendiendo adelante e propia mente es ioyel con perlas que las mugeres traen pendiente desdel cuello...'.

$\mathrm{N}$ 'por cadena o collar de oro'.

El interés de estas obras para la lingüística histórica española es también innegable. El Vocabulario, que ha sido una obra accesible, desde 1951, gracias a la reproducción que de ella hizo la Real Academia Española, y que además permite más fácilmente la búsqueda de las palabras castellanas, al estar casi ordenadas alfabéticamente, ha permitido su utilización en el Diccionario crítico etimológico de la lengua castellana y en el Diccionario crítico etimológico castellano e hispánico, de forma que en numerosas ocasiones tiene este Vocabulario la primera documentación de un vocablo o se contiene en él algún dato importante para entender su historia o explicar su etimología.

El Diccionario, en cambio, de más difícil manejo, poco accesible hasta hace algunos años, permitirá en su día completar muchos datos de finales de la Edad Media en los diccionarios históricos y etimológicos. Veamos algunos de ellos que no figuran en la nueva redacción del diccionario de Corominas o bien adelantan la documentación no solamente de esa magnífica obra (Corominas-Pascual, 1980-1983: DECH), sino también del Diccionario 
histórico de la lengua española (Real Academia Española, 1965-1972: D. hist.).

Abejaruco: D. hist.: s. f. ${ }^{14}$; $D E C H: 1513$.

Abominablemente: No figura ni en el $D$. hist. ni en el $D E C H$.

Abominador: $D$. hist.: 1726 ; no $D E C H$.

Abroquelado: D. hist.: 1593/1802; DECH : 1550 : abroquelar.

Acicalador: D. hist.: 1607 ; no $D E C H$.

Afeador: D. hist.: 1570 ; no $D E C H$.

Afeitadamente: $D$. hist.: 1565 ; no $D E C H$.

Aguage: D. hist.: 1535 ; $D E C H$ : s. f.

Ahornagamiento: $D$. hist.: 1570 ; no $D E C H$.

Alcançable $^{13}$ : no $D$. hist.; no $D E C H$.

Alcauetear: D. hist.: Santaella, Voc. ecles. (1523); DECH : 1589 : alcahuetar.

Allanamiento: D. hist.: G. Herrera, Agric. (1528); $D E C H$ : s. f.

Amansable: no $D$. hist.; no $D E C H$.

Amelgar: D. hist.: Santaella, Voc. ecles. (1523); DECH : 1550.

Animación: D. hist. $(1886,1901$...); DECH : s. f.

Aparejador: D. hist.: SigüenzA, Hist. ord. S. Jer. (1606); DECH: 1600.

Aperador: D. hist.: Espinel, Esc. Marcos de Obregón; DECH : 1618.

Apólogo: D. hist.: Cervantes, Quij.; P. Mejf́a (1547) ...; DECH: 1547.

Son tantos los vocablos que Nebrija proporciona a los historiadores de nuestro léxico que muchos de ellos no figuran no ya en una obra casi coetánea con la suya, como es la de Alfonso de Palencia (de 1490), sino incluso en otras tan posteriores como lo es el Tesoro de la lengua castellana $o$ española de Sebastián de Covarrubias, de 1611. A manera de ejemplo citaré unos cuantos términos que aparecen en nuestro gramático y que no se encuentran, en cambio, en los repertorios léxicos de A. de Palencia (1490: ed. 1967 y Hill, 1957) ni de S. de Covarrubias (1611, 1674: ed. 1979): aarón 'hierba':LE, EL; aballar:EL; abarcador:EL ; abarcadura:EL; abarcamiento:EL; abarraganamiento:LE, EL; abarramiento:EL; abarrancadero: EL; abarrar:EL ; abegera:EL ; abejaruco:LE ; abejuruco:LE, EL ; aben:EL;

14. $\mathrm{f} .=\sin$ fechar.

as Al no disponer de más tomos del $D$. hist. citado, continúo haciéndolo por el D. hist. publicado en 1933-1936: Real Academia Española, Diccionario histórico de la lengua española, Ed. Hernando, S. A., T. I: A, 1933; T. II : B-Cevilla, 1936, Madrid : D. hist. 
abispón:EL; ablandadura:EL; abollonado:EL; abollonar:EL; abominablemente:LE; abominador:LE; abonado:LE, EL; aborrible:EL; abortado:LE, EL; abortadura:EL; abotonadura:EL ... ${ }^{16}$.

Ante hechos como éstos no resulta en absoluto exagerado el juicio de Annamaria Gallina al caracterizar los diccionarios nebrisenses como "inesauribile miniera per i lessicografi di tutti i paesi" (1959: 102).

Para terminar quisiera recurrir de nuevo al léxico de la $a$ a la $a p$, a que antes he aludido, para señalar un hecho incidental, que. sin embargo, no carece de interés. Se trata de una particularidad que se ha relacionado con el andalucismo de Nebrija (Guitarte, 1974: 247-288) ${ }^{17}$. Sobre ello E. de Bustos ha dicho:

\footnotetext{
"Uno de los rasgos característicos del léxico andaluz, la particular abundancia de arabismos, no parece estar representado en el $V E L$ de modo especial” (1983: 219).
}

No es fácil estar de acuerdo con esta afirmación ${ }^{18}$ (Colón, 1982: 8689), sólo con comprobar los muchos arabismos que aparecen en los dos repertorios de Nebrija: alacrán:LE, EL; alardar:LE, EL; alambique:EL; alarde:LE, EL; alarifadgo:EL ; alarife:LE, EL; alatón:LE, EL ; alaxur:EL; albacea:EL; albahaca:LE, EL; albañi:LE, EL; albañar:EL; albañería:EL; albaquia:EL; albarda:LE, EL; albardán:LE; albardero:EL; albarrada:LE, EL ; albarrán:EL; albarrania:EL; albéitar:LE, EL; albeiteria:LE, EL; alberca:EL; albogue:EL; alboguero:EL; albórbolas:LE, EL; albornoz:LE, EL; alboroçar:EL; alboroço:EL; albricias:LE, EL; alcaçaba:EL; alcaçar: LE, EL; alcaduz:LE, EL; alcalde:LE, EL ; arcaduz:LE, EL, y muchos más.

Son éstas unas consideraciones sobre la obra lexicográfica de Nebrija que espero no resulten inoportunas. Son sólo el primer paso para una cuidadosa y lenta revisión de la obra lexicográfica del maestro salmantino.

\section{Marfa Lourdes Garcfa Macho.}

\footnotetext{
16 Otras variantes, derivados ... a veces sí aparecen en Covarrubias : abejoruco ... o en A. de Palencia : abominación ...

17 Personalmente no estoy totalmente de acuerdo con Guitarte respecto a lo que ha indicado sobre el andalucismo de Nebrija.

18 Ya en 1980, en el Congreso de Filología Románica, G. Colón sef́aló los muchos arabismos de la obra de Nebrija.
} 


\section{BIBLIOGRAFIA}

ACERo, IsAbel, 1985: "El Diccionario latino-español y el Vocabulario español-latino de Elio Antonio de Nebrija: análisis comparativo". Anuario de Lingüistica Hispánica, 1: 11-21. Valladolid.

Alonso, Amado, 1949: "Examen de las noticias de Nebrija sobre antigua pronunciación española". Nueva Revista de Filología Hispánica, 3 : 1-82. Méjico.

Bahner, Werner, 1966: La lingüistica española del Siglo de Oro. Madrid.

Bellido, José, 1945 : La patria de Nebrija. Noticia histórica. Madrid.

Bustos, Eugenio DE, 1983: "Nebrija, primer lingüista español". Academia Literaria Renacentista: Nebrija y la Introducción del Renacimiento en España, 3: 205-222. Salamanca.

Casares, Julio, 1947: “Nebrija y la Gramática castellana”. Boletin de la Real Academia Española, 26: 335-367. Madrid.

Colón, Germán, 1982: "Les llengües romàniques i llur selecció lexical". Actas del XVI Congreso Internacional de Lingüistica y Filología Románicas, 1: 86-89. Palma de Mallorca.

Colón, Germán, y Soberanas, Amadeu-J., ed., 1979: Antonio de Nebrija: Diccionario latino-español (1492). Estudio y edición: 9-36. Barcelona : Puvill.

Corominas, Joan, y Pascual, José A., 1980-1983 : Diccionario crítico etimológico castellano e hispánico. Madrid: Gredos. (DECH).

Covarrubias, Sebastián De, ed., 1979: Tesoro de la lengua castellana o española (1611, 1674). Madrid: Turner.

Gallina, Annamaria, 1959 : Contributi alla storia della lessicografia italo-spagnola dei secoli XVI e XVII. Firenze : Imp. Il Cenacolo.

Guitarte, Guillermo, 1974: "Alcance y sentido de las opiniones de Valdés sobre Nebrija". Estudios Filológicos y Lingüisticos. Homenajc a Angel Rosenblat en sus setenta años: 247-288. Caracas.

Hill, Joh N M., 1957 : Universal vocabulario de Alfonso de Palencia. Registro de voces españolas internas. Madrid: Real Academia Española: S. Aguirre, impresor.

LAPESA, RAFAeL, ed., 1980: Historia de la lengua española. Madrid: Gredos.

Menéndez Pidal, Ramón, 1950: "La Lengua en tiempo de los Reyes Católicos". Cuadernos Hispanoamericanos, 5 : 9-24. Madrid.

Nebrija, Elio Antonio. Vid Real Academia Española.

- Vid G. Colón y A. J. Soberanas.

Odriozola, Antonio, 1946: "La caracola del bibliófilo nebrisense". Revista de Bibliografía Nacional, $7:$ 3-114. Madrid.

Ormedo, Felix G., 1942: Nebrija (1441-1522). Debelador de la barbarie. Comentador eclesiástico. Pedagogo. Poeta. Madrid: Editora Nacional.

Palencia, Alfonso de, ed., 1967 : Universal vocabulario en latin y en romance (1490). Reproducción facsimilar de la edición de Sevilla. 2 tomos. Madrid: Gráficas Cóndor.

Quilis, Antonio, 1977 : Historia de la lengua española. I. Madrid: Universidad Nacional de Educación a Distancia.

- ed., 1980: Antonio de Nebrija: Gramática de la lengua castellana. Estudio y edición. Madrid : Editora Nacional.

Rral Academia Española, 1933-1936: Diccionario histórico de la lengua española. T. I y T. II. Madrid : Ed. Hernando, S. A. (D. hist.). 
- 1965-1972: Diccionario histórico de la lengua española. T. I y T. II. Madrid: Imp. Aguirre. (D. hist.).

, ed., 1951: Antonio de Nebrija: Vocabulario español-latino (1494?). Edición facsímil Real Academia Española. Madrid : Editora Castalia.

Rey, Alain, 1982: Encyclopédies et dictionnaires. Paris: Que sais-je?

Rosenblat, Angel, 1951: "Las ideas ortográficas de Bello", en Andrés Bello: estudios gramaticales, tomo V. Caracas. 